\title{
A novel online tool for capturing dietary and physical activity behaviours in adults: concurrent validity against standard reference methods
}

\author{
Frances Hillier, Alan Batterham, Sean Crooks and Carolyn Summerbell \\ University of Teesside, Middlesbrough, $U K$
}

Computer and online tools are a promising alternative to current standard methods for the assessment of dietary and physical activity behaviours in populations. The synchronised nutrition and activity program for adults (SNAPA) collects data on previous-day dietary and physical activity behaviours in adult populations. The aim of the present study was to evaluate the concurrent validity of SNAPA data against multiple-pass-recall (MPR) dietary interview and accelerometry data and, in addition, investigate the influence on the concurrent validity of using estimated portion sizes (EPS) $v$. average portion sizes (APS) ${ }^{(1)}$ during analysis of the SNAPA diet data.

Seventy-one adults (mean age 43.0 (SD 16.3) years) wore an accelerometer (Actigraph GT1M; Actigraph, Fort Walton Beach, FL, USA) during waking hours over a $24 \mathrm{~h}$ period. The following day participants completed SNAPA and a $24 \mathrm{~h}$ MPR interview. Percentage energy from fat; number of portions of fruit and vegetables; (F/V) and time spent (min) in moderate to vigorous physical activity (MVPA; accelerometry cut-point 2020 counts per min and estimated energy expenditure of $\geq 3$ metabolic equivalents (METS)) was calculated from data collected by SNAPA, MPR interviews and accelerometry for the same $24 \mathrm{~h}$ period.

\begin{tabular}{lccccccrrr}
\hline & \multicolumn{2}{c}{ SNAPA (EPS) } & & \multicolumn{2}{c}{ SNAPA (APS) } & & \multicolumn{2}{c}{ Reference method } \\
\cline { 2 - 3 } & Mean & SD & & Mean & SD & & Mean & SD \\
\hline Percentage energy from fat & 30.5 & 9.7 & & 32.3 & 9.8 & & 34.0 & 8.9 \\
No. of F/V portions & 3.3 & 2.9 & & 3.3 & 2.9 & & 4.9 & 3.8 \\
Minimum MVPA & 43.3 & 64.6 & & - & - & & 42.3 & 36.3 \\
\hline
\end{tabular}

Correlations between SNAPA and MPR-derived percentage energy from fat were 0.46 (bootstrapped 90\% CI 0.30, 0.60) using EPS and 0.48 (bootstrapped $90 \%$ CI $0.31,0.64$ ) using APS. The correlation between methods for portions of F/V was 0.42 (bootstrapped $90 \%$ CI $0.22,0.60)$ regardless of the portion size used during analysis. The correlation between SNAPA and accelerometry-derived MVPA was 0.39 (bootstrapped $90 \%$ CI $0.08,0.64$ ).

The results indicate reasonable concurrent validity of SNAPA $v$. the standard reference methods and suggest that the tool is suitable for the surveillance of dietary and physical activity behaviours in groups or populations. The use of EPS did not improve the concurrent validity of SNAPA for dietary behaviours compared with data collected by MPR interviews.

This study is part of a larger project commissioned by the Food Standards Agency.

1. Wrieden WL \& Barton KL (2006) Calculation and Collation of Typical Food Portion Sizes for Adults Aged $19-64$ and Older People Aged 65 and Over. Final Technical Report to the Food Standards Agency (Project N08026); available from Food Standards Agency Information Centre. 